\title{
Attitude Determination based on Common Reference Clock GPS/BDS Dual Antenna
}

\author{
Lei Zhang ${ }^{1,2}$, Wen Chen ${ }^{1,2}$, Chao $\mathrm{Yu}^{1,2}$, Danan Dong ${ }^{1,2}$ \\ 1. Engineering Center of SHMEC for Space Information and GNSS, East China Normal \\ University, Shanghai 200241, China \\ 2. Shanghai Key Laboratory of Multidimensional Information Processing, East China \\ Normal University, Shanghai 200241, China
}

\begin{abstract}
There is a technique innovation in GNSS receivers. That is to say, the common reference clock is adopted in more than two GNSS receivers and the carrier phase single difference can get the same technical effects as the double difference. This paper expresses in detail mathematical model method and some experiments, especially for GPS/BDS dual system. The related research work such as calculating precision of baseline and attitude, attitude determination and satellite elevation revealed the performance of GPS/BDS dual antenna of common reference clock.
\end{abstract}

Keywords: common reference clock, BDS, attitude determination

\section{Single Difference of the Common Clock reference dual antenna}

The Common reference clock means that the common crystal oscillator of the GNSS dual receiver sends the same pulse signal simultaneously. So, the GNSS dual receiver can realize to acquire data synchronously from the different antennas. One benefit of using a common clock reference is that the clock error is the same for all RF signals including carrier phase measurements, and thus it can be removed by forming single differences [1]. It is obvious that the single difference can be optimized and the accuracy of attitude determination can be further improved. Generally, the carrier phase observation equation of the common clock reference dual antenna is expressed as:

$$
\Phi_{i}=\frac{1}{\lambda}\left(\rho_{i}^{j}+c \cdot \delta \tau_{r_{i}}+c \cdot \delta \tau_{s}^{j}+T_{i}^{j}+I_{i}^{j}\right)+\varphi_{L B_{i}}+\varphi_{U P D_{i}}+\varphi_{M P_{i}^{j}}+N_{i}^{j}+\varepsilon(1)
$$

Where $\rho_{i}^{j}$ is the geometrical distance from GNSS satellite $j$ to GNSS receiver antenna $i, \delta \tau_{r_{i}}$ and $\delta \tau_{s}^{j}$ are the clock errors of GNSS receiver $i$ and GNSS satellite $j$ respectively, $\mathrm{T}_{i}^{j}$ and $\mathrm{I}_{i}^{\mathrm{j}}$ are respectively the atmospheric delay and ionospheric delay from GNSS satellite $\mathrm{j}$ to GNSS receiver antenna $\mathrm{i}$. And $\varphi_{\mathrm{LB}_{\mathrm{i}}}, \varphi_{\mathrm{UPD}_{\mathrm{i}}}$ and $\varphi_{\mathrm{MP}_{\mathrm{i}}^{\mathrm{j}}}$ are respectively the cable delay of GNSS receiver i, uncalibrated phase delay (UPD) of GNSS receiver and multi-path delay from GNSS satellite $j$ to GNSS receiver antenna $i . \lambda$ is the carrier wave length.c is the velocity of light. $\mathrm{N}_{\mathrm{i}}^{\mathrm{j}}$ is the ambiguity from GNSS satellite $\mathrm{j}$ to GNSS satellite $i$. eis measurement noise. And $i$ and $\mathrm{j}$ are natural numbers, e.g., 1, 2 and 3.[2]

In the case of the short baseline, single difference technique can only eliminate the errors including $\delta \tau_{s}^{j}, I_{i}^{j}$ and $\mathrm{T}_{\mathrm{i}}^{\mathrm{j}}$, but not eliminate $\delta \tau_{\mathrm{r}_{\mathrm{i}}}$. So, the double difference technique is the best way for the attitude determination. When the common clock reference is applied to GNSS receivers, it will take the advantage of clock synchronization. The common clock reference GNSS dual antenna as an example, the single difference equation can be optimized as followed:

$$
\Phi_{1}-\Phi_{2}=\Delta \Phi_{21}=\frac{1}{\lambda} \Delta \rho_{1-2}+\Delta \mathrm{N}_{1-2}+\Delta \mathrm{UPD}_{1-2}+\Delta \varepsilon_{1-2}
$$

The baseline vector $b_{12}$ is formed by connecting antenna 1 and antenna 2 . The baseline length will not generally exceed $10 \mathrm{~m}$, which is extremely short with respect to the distance between two satellitestations. It can be considered that the carrier signal is plane wave when the signal of GNSS satellitesS reaches two GNSS receivers, as shown in Figure 1. 


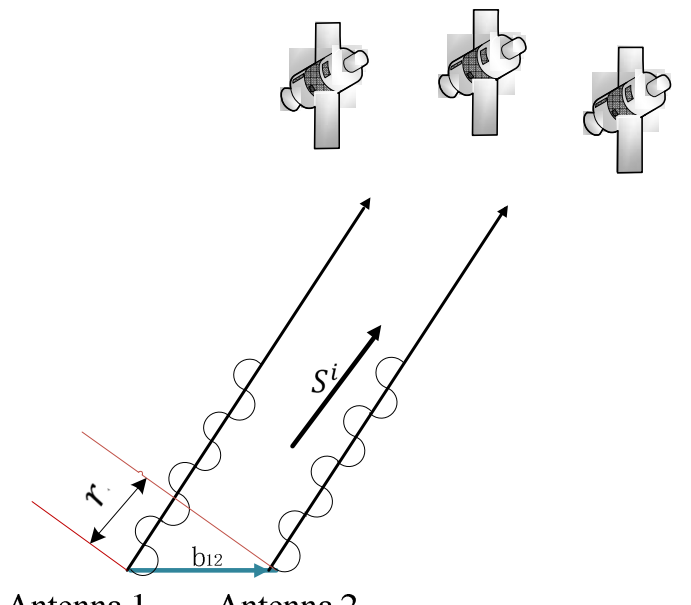

Antenna $1 \quad$ Antenna 2

Figure 1. Single difference model of dual antenna of common clock reference

The equation is:

$$
r=\left|b_{12}\right| \cdot \cos \alpha=b_{12} \cdot S^{i}
$$

Where $r$ is the distance difference from dual antenna to GNSS satellite. And $S^{i}$ is the unit vectorfrom dual antenna to GNSS satellite.

According to the equation (2) and the characteristics of common clock reference dual antenna, the observation error is neglected. So, the carrier phase single difference equation is expressed as follows:

$$
\Delta \phi_{21}^{\mathrm{S}}=\frac{\mathrm{f}}{\mathrm{c}}\left(\mathrm{R}_{1}^{\mathrm{S}}-\mathrm{R}_{2}^{\mathrm{S}}\right)-\left(\mathrm{N}_{1}^{\mathrm{S}}-\mathrm{N}_{2}^{\mathrm{S}}\right)=\frac{\mathrm{f}}{\mathrm{c}} \mathrm{S}^{\mathrm{i}} \cdot \mathrm{b}_{12}-\Delta \mathrm{N}_{21}^{\mathrm{S}}
$$

The equation (4) can be adjusted to:

$$
\lambda\left(\Delta \phi_{21}^{\mathrm{S}}+\Delta \mathrm{N}_{21}^{\mathrm{S}}\right)=\mathrm{S}^{\mathrm{i}} \cdot \mathrm{b}_{12}(5)
$$

Supposing there are n GNSS satellites in the sky, the equation (5) can be further adjusted to:

$$
\lambda\left[\begin{array}{c}
\Delta \phi_{21}^{1} \\
\Delta \phi_{21}^{2} \\
\cdots \\
\Delta \phi_{21}^{\mathrm{m}}
\end{array}\right]+\lambda\left[\begin{array}{c}
\Delta \mathrm{N}_{21}^{1} \\
\Delta \mathrm{N}_{21}^{2} \\
\cdots \\
\Delta \mathrm{N}_{21}^{\mathrm{m}}
\end{array}\right]=\left[\begin{array}{c}
\mathrm{S}^{1} \\
\mathrm{~S}^{2} \\
\cdots \\
S^{\mathrm{m}}
\end{array}\right] \cdot \mathrm{b}_{12} \quad(6)
$$

If the ambiguity of whole cycles is unknown, the baseline vector cannot be solved in case of the single epoch.That is to say, the attitude angle cannot be solved because of the correspondence of baseline vector in two coordinate system. As a result, it is crucial to fix the ambiguity of whole cycles when solving the carrier attitude. After solving the ambiguity of whole cycles, the baseline vector can be solved through equation (6)

\section{Related work}

\subsection{Basic problems of dual system}

While most clocks derive their time from Coordinated Universal Time (UTC), the atomic clocks on the satellites are set to GPS time (GPST for short). The difference is that GPS time is not corrected to match the rotation of the Earth, so it does not contain leap seconds or other corrections that are periodically added to UTC. The lack of corrections means that GPS time remains at a constant offset with International Atomic Time (TAI), and it is $\mathrm{T}_{\mathrm{ATI}}-\mathrm{T}_{\mathrm{GPST}}=19 \mathrm{~s}$. And as to BDS time system, the constant offset is $\mathrm{T}_{\mathrm{ATI}}-\mathrm{T}_{\mathrm{BDT}}=33 \mathrm{~s}$. In this paper, GPST is adopted as the reference time system.

The World Geodetic System of 1984 (WGS-84) is a 3-D, earth-centered reference system developed by the former U.S. Defense Mapping Agency now incorporated into a new agency, National Imagery and Mapping 
Agency (NIMA). It is the official GPS reference system. BDS adopts the China Geodetic Coordinate System 2000 (CGCS2000). For most applications the difference between CGCS2000 and WGS84 can be neglected. So, WGS-84 is introduced as the unified reference coordinate system in this paper, without considering the conversion of two coordinate systems.

It is widely adopted that the GNSS satellite position is calculated by making use of broadcast ephemeris. The GEO satellites of BDS are static relative to the earth. The characteristics of the BDS constellation is different to GPS. So, it needs the coordinate transformation.

\subsection{Correlation Analysis of Dual System Observation}

One of the dual antenna in the common reference clock GPS/BDS receiver system can be as research object. The carrier phase observation error of BDS is assumed to satisfy a normal distribution with the mean value of 0 and the variance of $\sigma_{\mathrm{B}}^{2}$. And the carrier phase observation error of GPS is Assumed to satisfy a normal distribution with the mean value of 0 and the variance of $\sigma_{G}^{2}$. [4] When there are $m$ BDS satellites and $n$ GPS satellites available, the covariance matrix of observation noise is expressed as followed:

$$
\begin{aligned}
& \operatorname{cov}\left(\phi_{i}^{B_{j}}\right)=\sigma_{B}^{2} \quad j=1 \ldots m, i=1,2 \\
& \quad \operatorname{cov}\left(\phi_{i}^{G_{j}}\right)=\sigma_{G}^{2} \quad j=1 \ldots n, i=1,2
\end{aligned}
$$

Where $\phi_{i}^{B_{j}}$ indicates that the carrier signal of BDS is received from BDS satellite jto antenna $i$, and $\phi_{i}^{G_{j}}$ indicates that the carrier signal of GPS is received from GPS satellite jto antenna $i$.

As to GPS satellite Gand BDS satellite Bof the common reference clock receiver in GPS/BDS dual system, carrier phase single difference observation equation of antenna 1 and antenna 2 is:

$$
\left\{\begin{array}{l}
\Delta \phi_{12}^{\mathrm{G}}=\phi_{2}^{\mathrm{G}}-\phi_{1}^{\mathrm{G}} \\
\Delta \phi_{12}^{\mathrm{B}}=\phi_{2}^{\mathrm{B}}-\phi_{1}^{\mathrm{B}}
\end{array}\right.
$$

And carrier phase difference observation of BDS is:

$$
\Delta \psi_{B}=\left[\begin{array}{c}
\Delta \phi_{12}^{\mathrm{B}_{1}} \\
\vdots \\
\Delta \phi_{12}^{\mathrm{B}_{\mathrm{m}}}
\end{array}\right]=\left[\begin{array}{ccccc}
1 & -1 & 0 & \cdots & 0 \\
\cdots & \cdots & \cdots & \cdots & \cdots \\
0 & 0 & \cdots & 1 & -1
\end{array}\right]\left[\begin{array}{c}
\phi_{2}^{\mathrm{B}_{1}} \\
\phi_{1}^{\mathrm{B}_{1}} \\
\vdots \\
\phi_{2}^{\mathrm{B}_{\mathrm{m}}} \\
\phi_{1}^{\mathrm{B}_{\mathrm{m}}}
\end{array}\right]
$$

According to covariance propagation, the covariance equation of observation noise is expressed as followed:

$$
\begin{array}{r}
\operatorname{cov}\left(\phi_{\mathrm{i}}^{\mathrm{B}_{\mathrm{p}}} \phi_{\mathrm{j}}^{\mathrm{B}_{\mathrm{q}}}\right)= \begin{cases}\sigma_{\mathrm{B}}^{2} & \mathrm{i}=\mathrm{j}, \mathrm{p}=\mathrm{q} \\
0 & \text { others }\end{cases} \\
\operatorname{cov}\left(\Delta \phi_{12}^{\mathrm{B}_{\mathrm{p}}} \Delta \phi_{12}^{\mathrm{B}_{\mathrm{q}}}\right)=\mathrm{E}\left(\Delta \phi_{12}^{\mathrm{B}_{\mathrm{p}}} \Delta \phi_{12}^{\mathrm{B}_{\mathrm{q}}}\right)-\mathrm{E}\left(\Delta \phi_{12}^{\mathrm{B}_{\mathrm{p}}}\right) \mathrm{E}\left(\Delta \phi_{12}^{\mathrm{B}_{\mathrm{q}}}\right)= \begin{cases}2 \sigma_{\mathrm{B}}^{2} & \mathrm{p}=\mathrm{q} \\
0 & \mathrm{p} \neq \mathrm{q}\end{cases}
\end{array}
$$

The singledifference observations of BDS are irrelevant from equation (12), and the covariance matrix is:

$$
\operatorname{cov}\left(\Delta \psi_{\mathrm{B}}\right)=\operatorname{cov}\left(\left[\begin{array}{c}
\Delta \phi_{12}^{\mathrm{B}_{1}} \\
\vdots \\
\Delta \phi_{12}^{\mathrm{B}_{\mathrm{m}}}
\end{array}\right]\right)=\left[\begin{array}{cccc}
2 & 0 & \cdots & 0 \\
0 & 2 & \cdots & 0 \\
\vdots & \vdots & \ddots & \vdots \\
0 & 0 & \cdots & 2
\end{array}\right] \sigma_{\mathrm{B}}^{2}
$$

Similarly, covariance matrix of the singledifference observations of GPS is:

$$
\operatorname{cov}\left(\Delta \Psi_{\mathrm{G}}\right)=\left[\begin{array}{cccc}
2 & 0 & \cdots & 0 \\
0 & 2 & \cdots & 0 \\
\vdots & \vdots & \ddots & \vdots \\
0 & 0 & \cdots & 2
\end{array}\right] \sigma_{\mathrm{G}}^{2}(14)
$$


So, covariance matrix of the single difference observations of GPS/BDS dual system is:

$$
\operatorname{cov}\left(\Delta \psi_{\mathrm{G}}, \Delta \psi_{\mathrm{B}}\right)=\left[\begin{array}{cc}
\operatorname{cov}\left(\Delta \psi_{\mathrm{G}}\right) & 0 \\
0 & \operatorname{cov}\left(\Delta \psi_{\mathrm{B}}\right)
\end{array}\right]
$$

Taking the first BDS satellite as the reference satellite, double difference observation of BDS is:

$$
\nabla \Delta \psi_{\mathrm{B}}=\left[\begin{array}{ccccc}
-1 & 1 & 0 & \cdots & 0 \\
\cdots & \cdots & \cdots & \cdots & \cdots \\
-1 & \cdots & 0 & 0 & 1
\end{array}\right]\left[\begin{array}{c}
\Delta \phi_{12}^{\mathrm{B}_{1}} \\
\Delta \phi_{12}^{\mathrm{B}_{2}} \\
\vdots \\
\Delta \phi_{12}^{\mathrm{B}_{\mathrm{m}}}
\end{array}\right]=\operatorname{Rcov}\left(\Delta \psi_{\mathrm{B}}\right)(16)
$$

Where $=\left[\begin{array}{ccccc}-1 & 1 & 0 & \cdots & 0 \\ \cdots & \cdots & \cdots & \cdots & \cdots \\ -1 & \cdots & 0 & 0 & 1\end{array}\right]$

According to error propagation:

$$
\operatorname{cov}\left(\nabla \Delta \psi_{\mathrm{B}}\right)=\operatorname{R} \operatorname{cov}\left(\Delta \psi_{\mathrm{B}}\right) \mathrm{R}^{\mathrm{T}}=2 \sigma_{\mathrm{B}}^{2}\left[\begin{array}{cccc}
2 & 1 & \cdots & 1 \\
1 & 2 & \cdots & 1 \\
\vdots & \vdots & 2 & 1 \\
1 & 1 & \cdots & 2
\end{array}\right]
$$

Similarly, double difference observation of GPS is:

$$
\operatorname{cov}\left(\nabla \Delta \Psi_{G}\right)=2 \sigma_{G}^{2}\left[\begin{array}{cccc}
2 & 1 & \cdots & 1 \\
1 & 2 & \cdots & 1 \\
\vdots & \vdots & 2 & 1 \\
1 & 1 & \cdots & 2
\end{array}\right]
$$

So, covariance matrix of the double difference observations of GPS/BDS dual system is:

$$
\operatorname{cov}\left(\nabla \Delta \psi_{\mathrm{G}}, \nabla \Delta \psi_{\mathrm{B}}\right)=\left[\begin{array}{cc}
\operatorname{cov}\left(\nabla \Delta \psi_{\mathrm{G}}\right) & 0 \\
0 & \operatorname{cov}\left(\nabla \Delta \psi_{\mathrm{B}}\right)
\end{array}\right]
$$

Through the analysis and research above, single difference observations are linear independent, and double difference observations have certain correlation. But linear independent characteristic of single difference observations is more beneficial to the subsequent solution to single difference ambiguity. Because of low observation noise, single difference observation model can improve the solving precise better than traditional double difference observation model.

\section{Experimental Results}

\subsection{Calculating precision of baseline and attitude}

There are dual antenna of the GNSS receiver on the rooftop. The baseline between the antennas is 5.003meters in length and the experimental GNSS receiver is TrimbleBD982, as shown in Figure 2. The TrimbleBD982 receiver collects BDS B1 and GPS L1 carrier wave data with time sampling interval of 1s and observation time of 2,880 epochs. Detection and reparation for cycle slip of the experimental carrier wave date by use of Kalman filtering algorithm, and the integer ambiguity resolution based on fixing single difference ambiguities are the core questions in the experimental data processing. In the experiment, the fixed interval $\mathrm{m}=30, \Delta=0.2, \sigma=0.01$ for GPS, and the fixed interval $\mathrm{m}=300, \Delta=0.2, \sigma=0.01$ for BDS, and $\mathrm{m}=30, \Delta=0.2$, $\sigma=0.01$ for GPS/BDS dual system. 


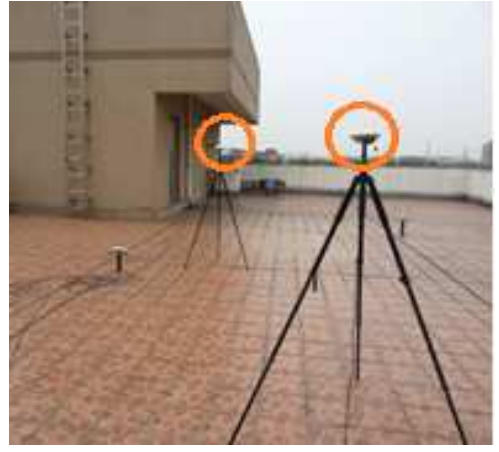

Figure 2.

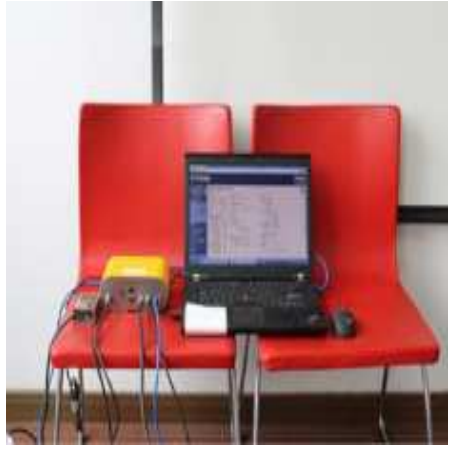

Experimental setup

In Figure 3, all the GREEN signal is resolution output result of dual system based on TrimbleBD982 receiver because the receiver collects data of GPS/BDS dual system. And RED, BLUE and BLACK signal illustrates the baseline calculating results for GPS, BDS and GPS/BDS dual system respectively.[5] Obviously, the baseline calculating precision and convergence effect of the experimental system is better than the result resolved by TrimbleBD982 receiver. As described in Table 1, the baseline standard deviations resolved based on GPS is $0.265 \mathrm{~mm}$, which is better than $0.276 \mathrm{~mm}$ based on BDS. And the baseline calculating precision reaches $0.227 \mathrm{~mm}$ by resolved based on GPS/BDS dual system. So, dual system helps to improve baseline calculating precision of GPS or BDS single system.
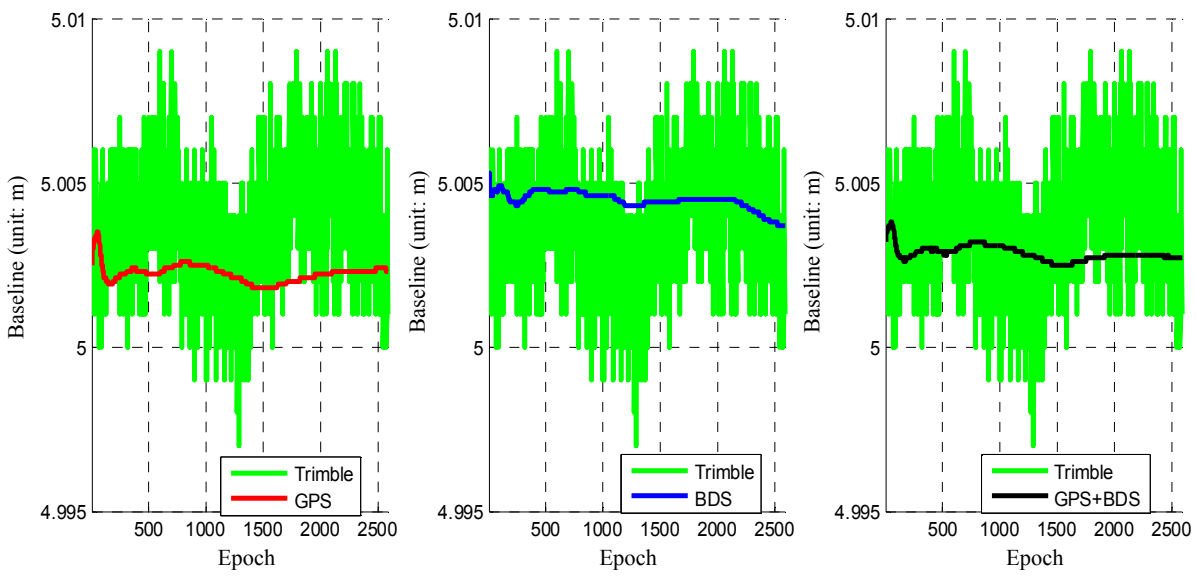

Figure 3. Baseline calculating results

Table1 Baseline calculating precision

\begin{tabular}{|c|c|c|}
\hline Satellite system & $\begin{array}{c}\text { Average baseline value } \\
(\mathrm{mm})\end{array}$ & $\begin{array}{c}\text { Standard deviation } \\
(\mathrm{mm})\end{array}$ \\
\hline GPS & 5.00263 & 0.265 \\
\hline BDS & 5.00446 & 0.276 \\
\hline GPS/BDS & 5.00285 & 0.227 \\
\hline
\end{tabular}

The yaw angle and pitch angle are calculated as shown in Figure 4, 5 and 6. And the statistics of yaw angle and pitch angle are listed at length in Table 2. The standard deviations of yaw angle resolved based on GPS is $0.003477^{\circ}$, which is better than $0.004082^{\circ}$ based on BDS. And The standard deviations of pitch angle resolved based on GPS is $0.010959^{\circ}$, which is better than $0.018419^{\circ}$ based on BDS. So, attitude determination results of resolved based on GPS is better than the results based on BDS. Compared with the results of resolved based on GPS, the calculating precise of yaw angle and pitch by resolved based on GPS/BDS dual system is enhanced by $24.96 \%$ and $17.52 \%$ respectively. Similarly, compared with the results of resolved based on BDS, the calculating precise of yaw angle and pitch by resolved based on GPS/BDS dual system is enhanced by $36.08 \%$ and $50.93 \%$ respectively. 

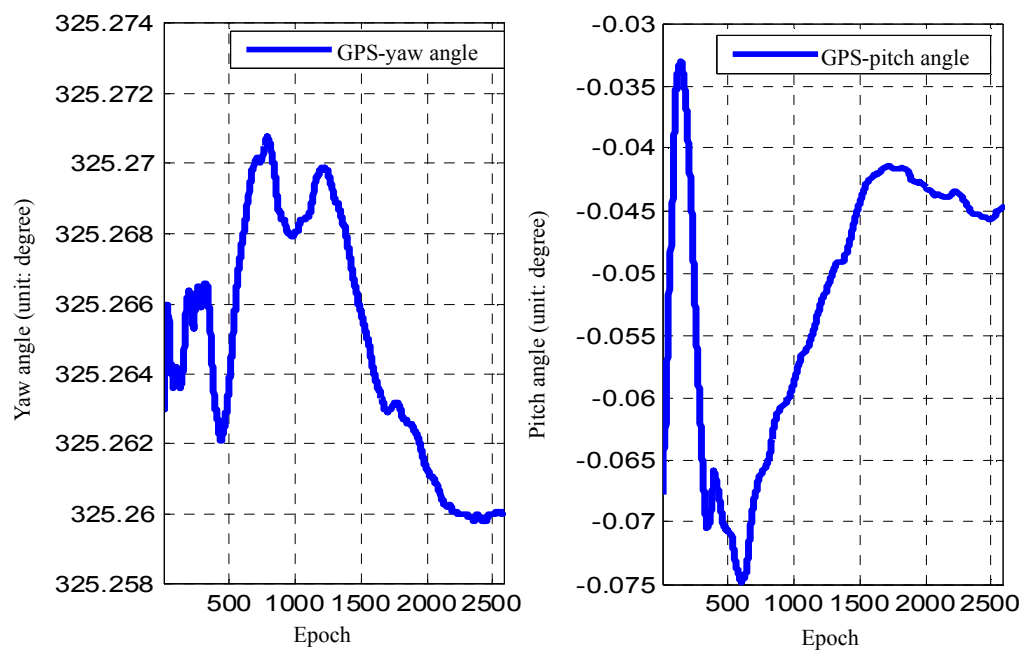

Figure 4. Yaw angle and pitch angle based on GPS
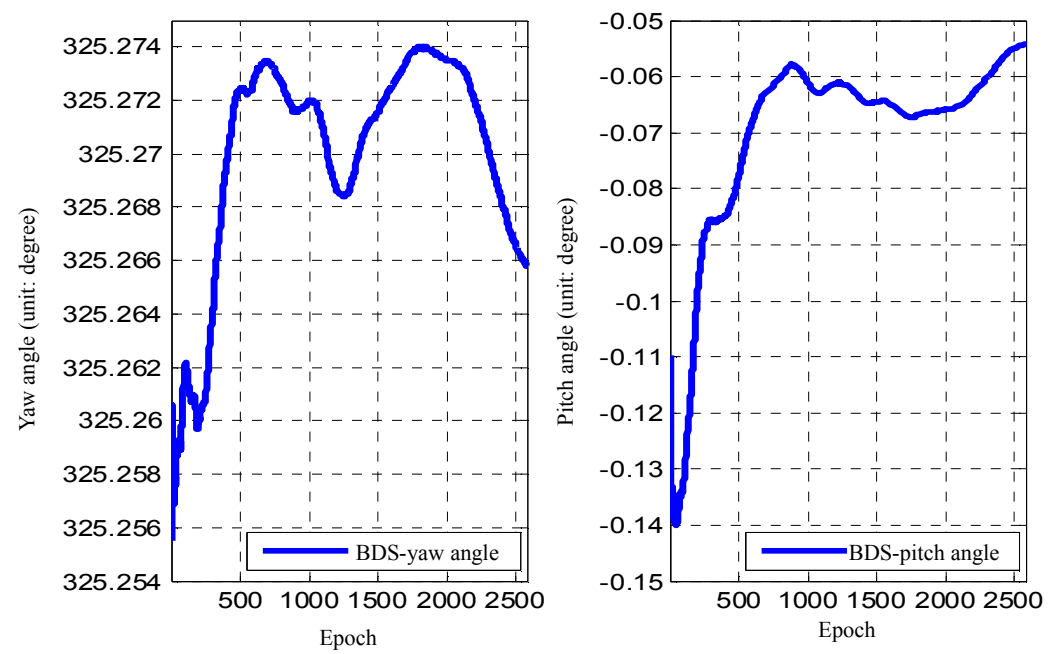

Figure 5. Yaw angle and pitch angle based on BDS
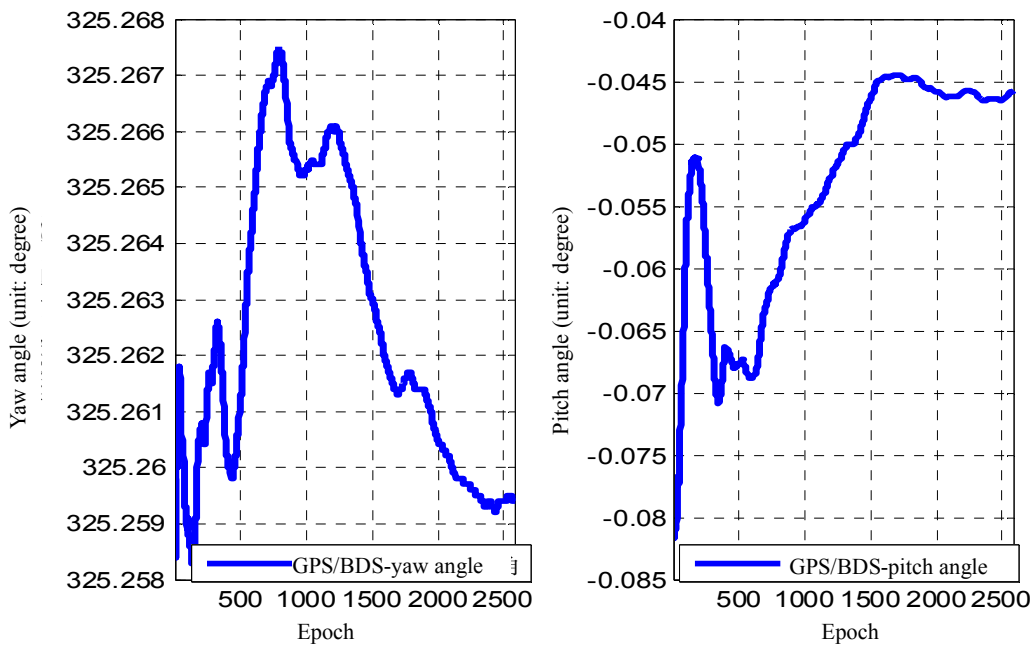

Figure 6. Yaw angle and pitch angle based on GPS/BDS 
Table2 Statistics of yaw angle and pitch angle

\begin{tabular}{|c|c|c|c|c|}
\hline $\begin{array}{c}\text { Satellite } \\
\text { system }\end{array}$ & $\begin{array}{c}\text { Average value of } \\
\text { yaw angle } \\
\text { (degree) }\end{array}$ & $\begin{array}{c}\text { Average value of } \\
\text { pitch angle } \\
\text { (degree) }\end{array}$ & $\begin{array}{c}\text { Standard deviation } \\
\text { of yaw angle } \\
\text { (degree) }\end{array}$ & $\begin{array}{c}\text { Standard deviation of } \\
\text { pitch angle } \\
\text { (degree) }\end{array}$ \\
\hline GPS & 325.2649 & -0.051859 & 0.003477 & 0.010959 \\
\hline BD & 325.270 & -0.070526 & 0.004082 & 0.018419 \\
\hline GPS/BD & 325.2624 & -0.053596 & 0.002609 & 0.009038 \\
\hline
\end{tabular}

\subsection{Attitude Determination and Satellite Elevation}

It takes place on the rooftop of the logistics building of Minhang Campus of East China Normal University on March 9, 2016. The multi-path effect is not serious because of high stories, large spatial spans and no upstairs covering. So, whether GNSS satellites are available or not only depends on cut-off elevation of GNSS satellites. The TrimbleBD982 receiver collects BDS B1 and GPS L1 carrier wave data with time sampling interval of $1 \mathrm{~s}$ and observation time of 2,880 epochs. As shown in Figure 7, the sky plots represent the relations between cut-off elevations and GPS or BDS satellites. The RED is for BDS satellites in the sky plots, and the GREEN is for GPS satellites. So, the number of available GNSS satellites at the angle of $10^{\circ}, 30^{\circ}, 45^{\circ}$ and $60^{\circ}$ respectively represents in Figure 8 . And the attitude angles on the four conditions of the different cut-off elevation are summarized in Table 3.
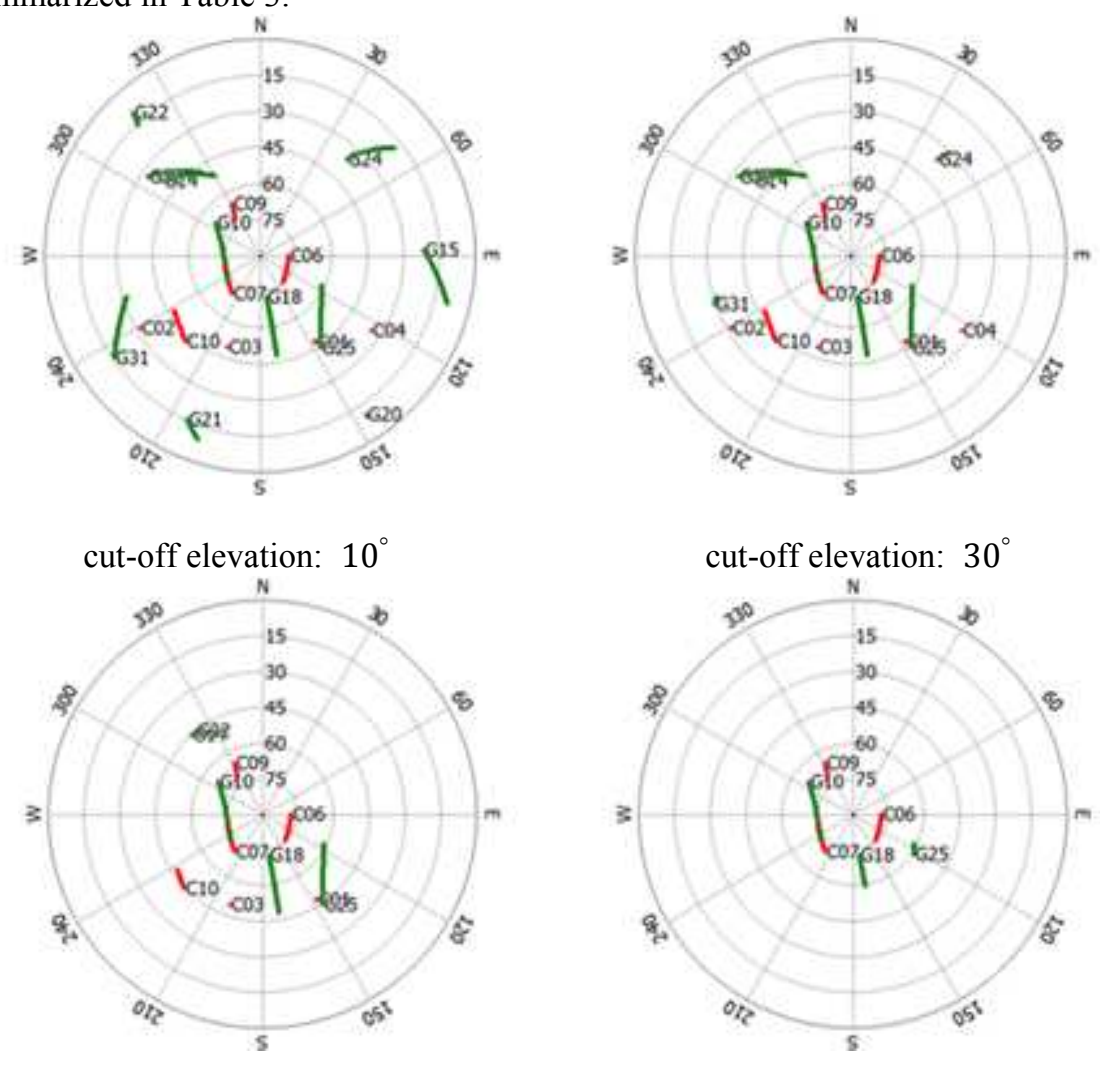

cut-off elevation: $45^{\circ}$

cut-off elevation: $60^{\circ}$

Figure 7. Sky plot at different cut-off elevation angles 

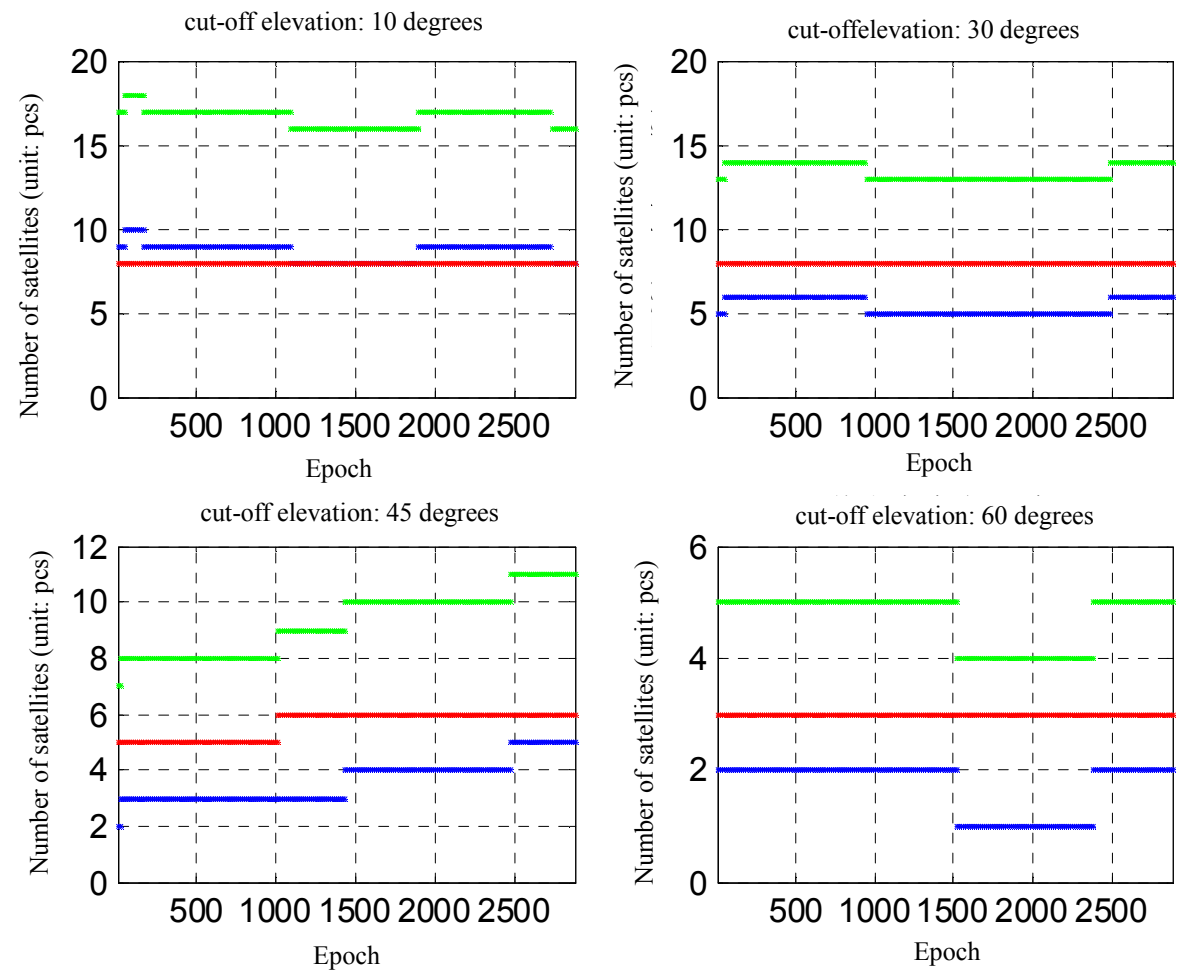

Figure 8. Number of available GNSS satellites on the four conditions

Table3 Attitude angles at different cut-off elevation

\begin{tabular}{|c|c|c|c|c|}
\hline \multicolumn{2}{|c|}{ Cut-off elevation angle } & GPS & BDS & GPS/BDS \\
\hline \multirow{2}{*}{$10^{\circ}$} & $\begin{array}{c}\text { Standard deviation of } \\
\text { yaw angle(degree) }\end{array}$ & 0.003859 & 0.007639 & 0.003343 \\
\hline & $\begin{array}{l}\text { Standard deviation of } \\
\text { pitch angle(degree) }\end{array}$ & 0.010002 & 0.013247 & 0.007197 \\
\hline \multirow{2}{*}{$30^{\circ}$} & $\begin{array}{c}\text { Standard deviation of } \\
\text { yaw angle(degree) }\end{array}$ & 0.004997 & 0.007639 & 0.003443 \\
\hline & $\begin{array}{l}\text { Standard deviation of } \\
\text { pitch angle(degree) }\end{array}$ & 0.09953 & 0.013247 & 0.011054 \\
\hline \multirow{2}{*}{$45^{\circ}$} & $\begin{array}{c}\text { Standard deviation of } \\
\text { yaw angle(degree) }\end{array}$ & 0.48993 & 0.09825 & 0.084407 \\
\hline & $\begin{array}{l}\text { Standard deviation of } \\
\text { pitch angle(degree) }\end{array}$ & 2.22506 & 0.21192 & 0.11044 \\
\hline \multirow{2}{*}{$60^{\circ}$} & $\begin{array}{c}\text { Standard deviation of } \\
\text { yaw angle(degree) }\end{array}$ & $*$ & 3.40627 & 0.27162 \\
\hline & $\begin{array}{l}\text { Standard deviation of } \\
\text { pitch angle(degree) }\end{array}$ & $*$ & 4.90121 & 0.44975 \\
\hline
\end{tabular}

Obviously, when cut-off elevation angle of GPS/BDS satellites is $10^{\circ}$, there are many available GPS/BDS satellites in the sky plot, and the determination precision of yaw and pitch angle for GPS or BDS is high. Especially, the determination precision of yaw and pitch angle for GPS/BDS dual system is higher than GPS or BDS single system. The number of GPS satellites decreases sharply along with elevation angle for GPS satellites increases. But, when cut-off elevation for BDS satellites is $30^{\circ}$, there are still 8 available satellites, and the determination precision of yaw and pitch angle remains the same as when cut-off elevation for BDS is $10^{\circ}$. When cut-off elevation for GPS/BDS is $45^{\circ}$, there are 5 available GPS satellites. But the resolution of GPS system is not realized at all because that epochs of PRN14 and PRN32 are few, and the epochs of 5 GPS satellites only occupy $14.1 \%$. So, all of these result in serious attitude determination. When cut-off elevation for GPS/BDS is $60^{\circ}$, there are less than 5 available GPS satellites and the attitude determination cannot be resolved. 
But there are still 3 IGSO satellites of BDS, and the attitude determination can preferably be resolved. Just then, there are still 5 satellites for GPS/BDS dual system, and standard deviation of yaw and pitch angle is $0.27162^{\circ}$ and $0.44975^{\circ}$ respectively.

\section{Conclusion}

High precision, reliability and integrality of GNSS depend on the number of available satellites and their geometrical distribution. BDS globalization is an important strategy to GNSS. Especially, IGSO and GEO satellites of BDS have a higher orbital altitude. There are enough available BDS satellites even in the harsh environment to ensure that the attitude precise can be improved and the measurement system can work continuously.

High performance, low cost and high accuracy are the main targets in the field of development on GNSS receivers. The algorithms of carrier phase single difference based on common clock reference dual antenna can fix single difference ambiguities without the enormous burden of doing extensive computations during the searching process. Traditionally, the algorithm of double difference cannot satisfy the attitude solution, when the number of available GNSS satellites are less than 4. But the single difference model can resolve it perfectly, especially for GPS/BDS dual system. The determination precision of attitude angles is significantly improved.

\section{Acknowledgment}

This work was partially supported by the Natural Science Foundation under grant No. 61671264. We gratefully acknowledge the invaluable contribution of the members of the laboratory and all the graduate students.

\section{References}

[1] Y. Li, K. Zhang, C. Roberts, M. Murata. On-the-fly GPS-based attitude determination using single- and double-differenced carrier phase measurements. GPS Solutions (2004) 8:93-102

[2] Chen W., Dong D., Cai M., et al. Short baseline solution from multi-antenna synchronized GNSS receiver and its applications for high-precision positioning . EGU General Assembly 2015

[3] M. Cai, W. Chen, F. Zhou, M. Wang, C. Yu. A partial GNSS ambiguity resolution for single-differenced carrier phase measurements. IUGG General Assembly 2015

[4] Zhou F, Dong D, Chen W, Cai M. A novel single-difference ambiguity fixing approach with multi-antenna synchronized BeiDou receiver. IUGG General Assembly 2015

[5] Minghua Wang, Danan Dong, Jiexian Wang, Wen Chen, Yanan Chen, Jingyang Lu.Multipath mitigation for short baseline based on dule-antenna clock-synchronized GNSS receiver. IUGG General Assembly 2015 